\title{
Adaptación e implementación del modelo de prevención de consumo de sustancias Planet Youth en Chile.
}

\section{Adaptation and implementation of the Planet Youth substance use prevention model in Chile}

Nicolás Libuy H. ${ }^{1,2}$, Carlos Ibáñez P. ${ }^{1,3}$, Viviana Guajardo T. ${ }^{1}$, Ana María Araneda F. ${ }^{1}$, Lorena Contreras E. ${ }^{1}$, Paula Donoso A. ${ }^{4,5}$, Adrian P. Mundt ${ }^{1,6}$.

\begin{abstract}
The prevalence of substance use is high among adolescents in several region around the world, specifically in Chile, and its prevention is an important public health challenge. We describe the adaptation and the feasibility to implement the Icelandic model of substance use prevention in adolescents "Planet Youth" in Chile as first experience in Latin America. This community prevention model focuses on the environment, culture and the promotion of health in adolescents, informed by local risk and protective factors. Implementation requires collaboration between academia and municipal authorities. Six municipalities of the Metropolitan Region, the University of Chile and the Icelandic Centre for Social Research and Analysis collaborated in the implementation of the Planet Youth model since 2018 in Chile. A substance use survey was translated, adapted, and applied to 7354 tenth grade students. The results were informed to schools and municipalities in order to work on modifications of the main risk and protective factors in their own community. In 2020, the prevention process has required some adaptation due to COVID-19 pandemic. We discuss sociocultural factors in the adaptation of this international prevention model transferred to Latin America. The implementation of the Planet Youth model is feasible in Chile and offers an opportunity to effectively prevent the substance use behaviors of adolescents in Latin America.
\end{abstract}

Key words: Prevention, Substance Use, Adolescents, Community based, Environmental prevention.

Rev Chil Neuro-Psiquiat 2021; 59 (1): 38-48

Los autores declaran no tener conflictos de intereses.

Aceptado: 2021/02/06

Recibido: 2021/01/16

Departamento de Psiquiatría y Salud Mental Norte, Hospital Clínico Universidad de Chile. Doctorado en Psicoterapia, Facultad de Medicina y Facultad de Ciencias Sociales, Universidad de Chile y Universidad Católica de Chile, Santiago, Chile.

Departamento de Neurociencia, Facultad de Medicina, Universidad de Chile Departamento Pediatría y Cirugía Infantil Oriente, Facultad de Medicina Universidad de Chile. SERJOVEN, Santiago, Chile

Facultad de Medicina, Universidad Diego Portales, Santiago, Chile 


\section{Introducción}

$\mathrm{E}^{\mathrm{I}}$ consumo de alcohol y drogas es un importante problema social y de salud en todo el mundo. El alcohol genera alta carga de enfermedad en años de vida perdidos por discapacidad y muerte ${ }^{1}$, mientras la marihuana es la droga más consumida a nivel mundial ${ }^{2}$. Las Américas es la región con la más alta prevalencia anual de consumo de marihuana con $8.8 \%$ en población entre 15 y 64 años ${ }^{2}$. Estados Unidos, Bolivia, Chile y Uruguay, han visto un aumento en el consumo de marihuana en los últimos años; además en Latinoamérica, la cocaína es la segunda droga por la que se busca tratamiento como sustancia principal ${ }^{2}$.

En Latino América, Chile tiene altas prevalencias de consumo de sustancias en adolescentes, con un consumo de alcohol del último año en escolares de $57 \%$, y de último mes de $31 \%$ donde el $62 \%$ de estos ellos ha bebido 5 o más tragos en una sola ocasión ${ }^{3}$. En cuanto a marihuana, la prevalencia de consumo en el último año subió de 14,8\% en 2001 a $30,9 \%$ en $2017^{3,4}$. Mientras en el mundo, el 4,7\% de los adolescentes entre 15 y 16 años ha consumido marihuana el último año ${ }^{2}$. Por sus prevalencias y consecuencias asociadas, el consumo de alcohol y otras sustancias genera un alto costo social y a la salud ${ }^{5}$.

La adolescencia es el periodo de la vida en que más frecuentemente se inicia el consumo de sustancias ${ }^{6,7}$. En esta etapa hay gran vulnerabilidad a la experimentación, mayor riesgo de trastornos por uso de sustancias, efectos más severos y mayor sensibilidad a la neurotoxicidad ${ }^{8}$. Debido a las altas prevalencias de consumo de sustancias en adolescentes y al impacto que estas tienen en una etapa de alta vulnerabilidad, es importante prevenir el consumo en adolescentes ${ }^{9}$. La identificación y el abordaje de los factores de riesgo y protectores más relevantes para el consumo permite planificar estrategias de prevención apropiadas ${ }^{10}$, sin embargo, estos factores pueden variar en distintas sociedades, países e incluso entre comunidades en un mismo país, de acuerdo a diferentes determinantes socioculturales. Por lo tanto, un abordaje local y adaptado a las necesidades de una comunidad específica debe ser considerado en la prevención.

Este artículo tiene por objetivo describir un modelo de prevención comunitaria desarrollado en Islandia, y la experiencia inicial del proceso de adaptación y factibilidad de implementación en seis comunas de Chile.

\section{Modelo Islandés}

Islandia ha llevado a cabo un proceso de prevención del consumo de sustancias en adolescentes abordando factores de riesgo y protección específicos basado en datos locales, y de esta manera han propuesto un modelo de prevención comunitario ${ }^{11}$. El Icelandic Centre for Social Research and Analysis (ICSRA) realiza una encuesta de bienestar juvenil anualmente desde los años 90, 1lamada "Youth in Iceland", que aborda el consumo de sustancias, explora factores protectores como apoyo, monitoreo y comunicación parental, bienestar escolar y participación en actividades extracurriculares tales como actividad deportiva organizada, y factores de riesgo como tiempo libre no supervisado, estilos de vida asociado a fiestas, y consumo en pares, entre otros ${ }^{12,13}$. Entre los factores protectores abordados en Islandia para la prevención primaria a nivel parental se incluyen: que los padres sepan con quién y dónde se encuentran sus hijos y que conozcan a los amigos de sus hijos y a sus respectivos padres. Además, resulta protectora la participación de los adolescentes en actividades deportivas organizadas, mientras un estilo de vida relacionado a fiestas es un factor de riesgo ${ }^{14}$. ICSRA ha diseminado el modelo de prevención realizado en Islandia a distintos lugares del mundo, llamándole Planet Youth 
(ver en https://planetyouth.org/).

Recientemente, el equipo de ICSRA ${ }^{15,16}$ ha publicado 5 principios guía del modelo islandés (Tabla 1) y 10 etapas (Tabla 2) para la implementación de este modelo de prevención.

Tabla 1. Principios Guía Modelo Islandés Planet Youth

\begin{tabular}{|l|}
\hline \multicolumn{1}{|c|}{ Principios Guía Modelo Islandés } \\
\hline 1. Aplicar un enfoque de prevención primaria diseñado para mejorar el ambiente social. \\
\hline $\begin{array}{l}\text { 2. Enfatizar la acción comunitaria y aprovechar los colegios como el centro natural de los } \\
\text { esfuerzos de la comunidad para apoyar la salud, aprendizaje y una vida exitosa de los niños y } \\
\text { adolescentes. }\end{array}$ \\
\hline $\begin{array}{l}\text { 3. Involucrar y empoderar a los miembros de la comunidad en la toma de decisiones prácticas } \\
\text { usando datos y diagnósticos locales, accesibles y de alta calidad. }\end{array}$ \\
\hline $\begin{array}{l}\text { 4. Integrar investigadores, políticos, profesionales y miembros de la comunidad en un equipo } \\
\text { unificado dedicado a resolver problemas complejos del mundo real. }\end{array}$ \\
\hline $\begin{array}{l}\text { 5. Hacer coincidir el alcance de la solución con el ámbito del problema, incluyendo interven- } \\
\text { ciones con énfasis de largo plazo y esfuerzos para organizar adecuadamente los recursos de la } \\
\text { comunidad. }\end{array}$ \\
\hline
\end{tabular}

Tabla 2. Etapas Modelo Islandés Planet Youth

\begin{tabular}{|l|}
\hline \multicolumn{1}{|c|}{ Etapas Modelo Islandés } \\
\hline Etapa 1: Identificación del equipo local, desarrollo y construcción de capacidad \\
\hline Etapa 2: Identificación de fondos locales, desarrollo y construcción de capacidad \\
\hline Etapa 3: Planificación de la recolección de datos e involucramiento de la comunidad \\
\hline Etapa 4: Recolección de datos, procesamiento, incluyendo diagnósticos basados en los datos \\
\hline Etapa 5: Fortaleciendo la participación de la comunidad y el involucramiento \\
\hline Etapa 6: Diseminación de los resultados \\
\hline $\begin{array}{l}\text { Etapa 7: Establecimiento de objetivos de la comunidad y otras respuestas organizadas frente } \\
\text { a los resultados }\end{array}$ \\
\hline
\end{tabular}




\section{Planet Youth en Chile}

En 2018 se inició una colaboración entre seis municipalidades (Colina, Las Condes, Lo Barnechea, Melipilla, Peñalolén y Renca), ICSRA y la Universidad de Chile para implementar el modelo Planet Youth.

La estrategia de prevención basada en el modelo de Islandia, Planet Youth en Chile (en adelante, PY) está dirigida a toda la población adolescente de cada comuna vinculada al sistema educativo formal. Para evaluar el consumo de sustancias en adolescentes, se planificó realizar encuestas de corte transversal a estudiantes de educación secundaria en décimo grado $\left(2^{\circ}\right.$ año de educación media en Chile), cada 2 años.

En el proceso de implementación y adaptación del modelo PY en las seis comunas involucradas, se ha coordinado la participación de tres partes: ICSRA, cada Municipalidad y la Universidad de Chile. Cada Municipalidad definió un responsable y un equipo de prevención local. El equipo de la Universidad de Chile se ha encargado de llevar a cabo los procesos de adaptación del modelo, proveer de apoyo técnico a cada comuna y de la coordinación entre ICSRA y cada Municipalidad, para facilitar la gestión de implementación del modelo PY.

Se firmaron memorandos de entendimiento internacionales entre cada municipio, ICSRA y la Universidad de Chile. Esta colaboración definió un plan de cinco años con la aplicación de las encuestas PY cada dos años, y entrega de informes de resultados a colegios y municipios con recomendaciones desde ICSRA para orientar la implementación local del plan comunitario de prevención PY. Este modelo no ofrece intervenciones estructuradas, ni manualizadas, pero tiene principios y recomendaciones para las municipalidades que la implementan, por tanto, las estrategias y actividades preventivas específicas se aplican según las definiciones de cada municipio.

\section{Encuestas}

Entre enero y marzo del 2018 el equipo coordinador de la Universidad de Chile tradujo la encuesta original de ICSRA. Se realizó una revisión de contenidos por parte de los equipos comunales, y una revisión por expertos de la Universidad de Chile. Se validó lingüística y semánticamente la adaptación de la encuesta en tres adolescentes chilenos voluntarios pertenecientes al mismo grupo etario de la población objetivo, que asisten a un centro de salud mental adolescente (SERJOVEN). La versión final aprobada por ICSRA y la Universidad de Chile contiene 77 preguntas. Se ejecutó el proceso de impresión de cuestionarios, embalaje, distribución y recolección, de acuerdo a lo establecido por ICSRA ${ }^{13}$.

La encuesta permite entregar un diagnóstico a cada comuna y colegio sobre el consumo de sustancias de los escolares y los factores relacionados al consumo. Desde cada municipio se contactó a los colegios particulares y municipales de la comuna para invitarlos a participar voluntariamente. Se confeccionó un consentimiento informado pasivo para ser enviado a los padres, a través del cual aquellos padres que rechazaban participar lo hacían de manera explícita. Además, se aplicó un documento de asentimiento para los alumnos, pudiendo aceptar o rechazar participar libremente si así lo decidían. Tanto el documento de consentimiento como el asentimiento fueron aprobados por el comité de ética del Hospital Clínico de la Universidad de Chile (OAIC 981/18).

Se conformaron equipos municipales encargados de implementar la encuesta en cada comuna, en coordinación con el equipo de la Universidad de Chile. Se capacitó a los equipos municipales sobre el procedimiento de aplicación de la encuesta PY en los colegios, quienes a su vez capacitaron a profesores de cada colegio. Se desarrollaron documentos de registro para sistematizar el proceso de 
aplicación de encuestas, realizar seguimiento y controlar el proceso. La encuesta fue aplicada la misma semana de junio de 2018 en los colegios participantes de las seis comunas.

\section{Prevención local}

Luego de 4 meses de aplicada la encuesta, ICSRA entregó informes con los resultados a municipalidades y colegios, junto con la base de datos anonimizada a cada municipalidad.

En 2018 participaron 7354 escolares de 117 colegios, con una tasa de respuesta de $86,9 \%$, siendo el $51,4 \%$ hombres y $48,6 \%$ mujeres.

Desde el equipo de la Universidad de Chile se apoyo a las comunas en el proceso de prevención en base a los informes de resultados y las recomendaciones realizadas por ICSRA, y se desarrollaron las siguientes herramientas para el trabajo con las comunas:

-Definición de objetivos transversales, comunes entre las seis comunas, considerando sus diferencias socioculturales y de gestión: 1. Reforzar la capacidad de gestión comunitaria, administrativa y política para la prevención a nivel comunal. 2. Disminuir el acceso al alcohol y a otras drogas a menores. 3. Aumentar el involucramiento parental y disminuir la normalización del consumo de alcohol y otras drogas en menores entre los padres y apoderados. 4. Promover un enriquecimiento ambiental a través de actividades recreativas extracurriculares organizadas.

-Utilización de Marco Lógico: se propuso diseñar el plan de prevención en base a la metodología de Marco Lógico que permite planificar, seguir y evaluar programas ${ }^{17}$.

-Índice de prevención comunal: Se tradujo y adaptó un instrumento sueco ${ }^{18}$ de monitoreo del nivel de desarrollo de la prevención local en 5 dimensiones: personal y presupuesto, política de prevención, colaboración, patentes de alcohol y fiscalización, y actividades de prevención. Este instrumento brinda una medida simple para orientar el trabajo preventivo y es comparable entre municipios.

-Compromiso Parental: Se tradujo y adaptó un documento desarrollado en Islandia con recomendaciones para promover relaciones positivas entre padres e hijos, aumentar la participación en actividades escolares y recreativas, y establecer límites claros en la crianza (3 formatos distintos para estudiantes de $1^{\circ}$ a $5^{\circ}$ básico, de $6^{\circ}$ a $8^{\circ}$ básico y de $1^{\mathrm{o}}$ a $4^{\circ}$ medio). Además, se incorporó una propuesta metodológica para implementar esta herramienta en establecimientos educacionales.

Además de estas herramientas, y de las estrategias adoptadas por las comunas basadas en las recomendaciones realizadas por ICSRA, cada municipio desarrollo actividades específicas ajustadas a su realidad y contexto, dentro de los principios del modelo PY. Así mismo, el marco general y principios del modelo comunitario de prevención PY, permitieron que los distintos actores en prevención de las comunas participantes pudieran organizar su trabajo de forma coordinada, incluyendo actividades de prevención que se venían realizando previamente y agregando nuevas estrategias y actividades centradas en las necesidades identificadas y priorizadas, en un trabajo flexible, dirigido por cada comuna.

En 2020, debido a la pandemia por COVID-19, la encuesta fue adaptada para ser aplicada en formato online, en una versión abreviada e incorporando preguntas sobre la pandemia y su impacto en los participantes. En noviembre de 2020 participaron las mismas seis comunas en la encuesta, con un total de 5528 encuestas con una tasa de respuesta promedio por colegio de $72,8 \%$ en 96 colegios. Los resultados son informados a cada colegio y comuna en reportes preparados por ICSRA para orientar las próximas 
estrategias de prevención.

\section{Discusión}

El proceso de implementación del modelo Planet Youth en seis comunas se está ejecutando con el objetivo de disminuir el consumo de sustancias en adolescentes en un plan que se encuentra actualmente en progreso, siendo una experiencia única de prevención del consumo de sustancias en adolescentes para Chile, ya que abarca una gran población a través de las municipalidades, y ha exigido una estrecha colaboración y coordinación entre: autoridades políticas y administrativas de cada comuna, los equipos de profesionales en prevención que llevan a cabo el trabajo con la comunidad y la academia con las orientaciones desde los equipos de la Universidad de Chile y de ICSRA. De acuerdo con esta experiencia que hemos reportado, parece promisoria la adaptación de este modelo de prevención basado en evidencia internacional para implementarlo en países de Latinoamérica.

Debido a que el modelo PY no ofrece intervenciones estructuradas, desarrollar y adaptar herramientas de apoyo para la prevención local según las necesidades específicas de las municipalidades ha permitido estructurar la implementación de la prevención, y sirve para apoyar a equipos comunales.

El modelo de prevención islandés está enfocado en la modificación del ambiente social a través del involucramiento comunitario en función de un diagnóstico local del consumo en adolescentes y sus factores asociados. El modelo se basa en la retroalimentación de datos locales sobre el consumo de adolescentes a las comunidades para que se involucren y modifiquen los factores de riesgo y protectores identificados. Un aspecto de especial relevancia en la implementación descrita en la experiencia chilena ha sido el rol de la autoridad comunal en el abordaje de esta temática y cómo gracias a ese compromiso se han estructurado mesas de trabajo intersectoriales y colaboración entre distintas unidades municipales y otros actores relevantes con un objetivo común.

Uno de los principales desafíos implicados en este proceso se relaciona con la transferencia de un modelo que ha mostrado efectividad en un contexto sociocultural diferente, proveniente de un país con características distintas. En Chile, comunas con diferentes características socioeconómicas están llevando a cabo este modelo, lo que resulta de especial importancia considerando la gran variabilidad en el acceso a recursos que existe entre las distintas comunas en nuestro país, y como esto puede afectar una implementación exitosa de la experiencia islandesa en un contexto diferente.

Algunas estrategias recomendadas por ICSRA están fuertemente centradas en el uso del tiempo libre y en el rol de los padres en la crianza de sus hijos. Con relación al desarrollo de actividades libres de consumo, Bickel W et al, ${ }^{19}$, han planteado que, de acuerdo con la teoría de conducta económica, alternativas de refuerzo que compiten con el consumo de sustancias aumentan el costo de oportunidad del consumo de sustancias, disminuyendo el consumo, por lo tanto, de manera inversa, las personas con pocas alternativas al uso de sustancias tienen menos posibilidades de cambiar su consumo exitosamente. Ofrecer actividades alternativas al consumo que estén libres de alcohol y droga en los cuales las personas se involucren, participen, se motiven y las disfruten, actuarían como actividades reforzadoras que disminuyen la probabilidad de consumo de sustancias ${ }^{20}$.

En cuanto a la parentalidad, los estilos parentales autoritativos (alta expectativa, reglas claras, y apoyo) son protectores; mientras padres poco involucrados tendrían adolescentes más propensos a consumir 10,21. Así mismo, los pares y familiares influyen 
de manera importante en lo que es considerado como normativo, saludable y aceptable para los adolescentes ${ }^{10,22}$, y por consiguiente influyen en las conductas de consumo. En el contexto de cambios sociales y legales sobre el consumo de marihuana, los padres y equipos de salud se enfrentan a nuevo desafío con los adolescentes sobre como discutir y establecer reglas sobre el consumo ${ }^{10}$, 23. El bajo rechazo parental de consumo de marihuana se ha asociado a un aumento del consumo en escolares chilenos ${ }^{4}$.

En el caso de Chile, se ha descrito que las normas familiares podrían ser más importantes que las sociales para las personas ${ }^{24}$. Esto está en línea con la importancia de la parentalidad que ha sido descrita en población hispánica cuando se la compara con caucá$\operatorname{sicos}^{25}$. Por lo tanto, un enfoque familiar robusto, orientado a fomentar una parentalidad adecuada e involucrada podría resultar más apropiado a esta cultura que las normas y el control social.

Sin duda, los aspectos sociodemográficos y económicos, las capacidades de las municipalidades de fomentar el buen uso del tiempo libre en jóvenes, y factores de parentalidad, están relacionados con aspectos socioculturales. Para la implementación y adaptación apropiada de experiencias internacionales se debe tener en cuenta algunos factores socioculturales que influyen sobre el consumo de sustancias y por lo tanto sobre la prevención ${ }^{26}$. Las estrategias basadas en evidencia internacional deben ser transferidas de manera apropiada al contexto local. Además de lo mencionado, deben incorporarse otros factores socioculturales locales en el proceso de adaptación, como la propia opinión, participación y experiencia de los involucrados y de los profesionales que trabajan en prevención en el territorio. Una adecuada adaptación de las estrategias de prevención podría ofrecer mejores resultados, mayor aceptabilidad y menores dificultades en el momento de la implementación, tal como ha sido descrito en el caso de otras dos intervenciones preventivas culturalmente adaptadas para latinos (keepin' it REAL y Families Preparing the New Generation) en Estados Unidos y en México ${ }^{27}$ 28. Otras experiencias de programas preventivos adaptados culturalmente son el programa Habilidades para la vida ${ }^{29,30}$, Familias unidas ${ }^{31}$ y Comunidades que se cuidan ${ }^{32,33}$.

La magnitud del problema de consumo de sustancias en adolescentes, sus costos y consecuencias, exigen levantar evidencia científica sobre prevención del consumo de sustancias en el contexto latinoamericano, ya que la mayoría de la investigación proviene en general de países de altos ingresos ${ }^{34,35}$. Las diferencias de contexto sociocultural hacen necesario fortalecer y desarrollar la investigación en los lugares donde requieren ser aplicadas. El modelo PY puede ofrecer una importante oportunidad para prevenir el consumo de sustancias en jóvenes de manera efectiva, y su implementación piloto en seis comunas en Chile ha generado interés también en otros países de Latinoamérica.

Financiamiento:

\section{-Proyecto FONIS SA19I0152, CONICYT ANID}

-Proyecto apoyado en la ejecución por la I. Municipalidad de Colina, I. Municipalidad de Las Condes, I. Municipalidad de Lo Barnechea, I. Municipalidad de Melipilla, I. Municipalidad de Peñalolén, e I. Municipalidad de Renca.

-Nicolás Libuy ha sido financiado con beca ANID CONICYT PFCHA/DOCTORADO NACIONAL/2018 - 21180520. 
Resumen

El consumo de sustancias en adolescentes es altamente prevalente en varias regiones del mundo, y especialmente en Chile, siendo su prevención un importante desafio para la salud pública. Este artículo describe el modelo islandés de prevención del consumo de sustancias en adolescentes "Planet Youth", su adaptación y factibilidad de implementación en Chile, como primera experiencia en Latinoamérica. Este modelo comunitario está enfocado en la prevención ambiental y en la promoción de la salud de niños, niñas y adolescentes, basado en un diagnóstico local y oportuno de factores protectores y de riesgo, con colaboración de la autoridad local y la academia. Seis comunas de la región metropolitana en colaboración con la Universidad de Chile y el Icelandic Centre for Social Research and Analysis inician su implementación en 2018. Se tradujo y adaptó la encuesta islandesa que fue aplicada a 7354 estudiantes de $2^{\circ}$ medio, cuyos resultados se retroalimentaron a colegios y municipalidades para trabajar en la modificación de los principales factores de riesgo y protección. En 2020, el proceso ha requerido algunas adaptaciones debido a la pandemia por COVID-19. Se discute acerca de factores socioculturales relevantes en la adaptación de estrategias basadas en evidencia internacional que se transfieren a un país diferente. La implementación del modelo Planet Youth es factible en Chile y ofrece una importante oportunidad para prevenir el consumo de sustancias en jóvenes de manera efectiva en Latinoamérica.

Palabras clave: Prevención, Consumo de sustancias, Adolescentes, Comunitaria, Prevención ambiental.

\section{Referencias Bibliográficas}

1.- Stanaway, J. D., Afshin, A., Gakidou, E., Lim, S. S., Abate, D., Abate, K. H., ... Murray, C. J. L. (2018). Global, regional, and national comparative risk assessment of 84 behavioural, environmental and occupational, and metabolic risks or clusters of risks for 195 countries and territories, 19902017: A systematic analysis for the Global Burden of Disease Stu. The Lancet, 392(10159), 1923-1994.

2.- United Nations Office on Drugs and Crime. (2020). Booklet 2: Drug Use and Health Consequences. World Drug Report.

3.- Servicio Nacional para la Prevención y Rehabilitación del Consumo de Drogas y Alcohol, S. (2018). Décimo Se- gundo Estudio Nacional de Drogas en Población Escolar de Chile, 2017 80 Básico a 4o Medio. SENDA Ministerio del Interior y Seguridad Pública Gobierno de Chile.

4.- Libuy, N., Ibáñez, C., \& Mundt, A. P. (2020). Factors related to an increase of cannabis use among adolescents in Chile: National school based surveys between 2003 and 2017. Addictive Behaviors Reports, 11(October 2019), 100260.

5.- Servicio Nacional para la Prevención y Rehabilitación del Consumo de Drogas y Alcohol, S., \& Pontificia Universidad Católica de Chile, P. (2018). Estudio del Costo Económico y Social del Consumo de Alcohol en Chile. Departamento de Salud Pública Universidad Católica de Chile, 161. Retrie- 
ved from https://www.infodrogas.org/ drogas/alcohol? showall=\&start $=2$

6.- Degenhardt, L., Chiu, W. T., Sampson, N., Kessler, R. C., Anthony, J. C., Angermeyer, M., ... Wells, J. E. (2008). Toward a global view of alcohol, tobacco, cannabis, and cocaine use: Findings from the WHO Degenhardt, L., Chiu, W. T., Sampson, N., Kessler, R. C., Anthony, J. C., Angermeyer, M., ... Wells, J. E. (2008). Toward a global view of alcohol, tobacco, cannabis,. PLoS Medicine, 5(7), 1053-1067

7.- Merikangas, K. R., He, J. P., Burstein, M., Swanson, S. A., Avenevoli, S., Cui, L., ... Swendsen, J. (2010). Lifetime prevalence of mental disorders in U.S. adolescents: Results from the national comorbidity survey replication-adolescent supplement (NCS-A). Journal of the American Academy of Child and Adolescent Psychiatry, 49(10), 980 989.

8.- Koob, G. F., \& Volkow, N. D. (2016). Neurobiology of addiction: a neurocircuitry analysis. The Lancet Psychiatry, 3(8), 760-773.

9.- Degenhardt, L., Stockings, E., Patton, G., Hall, W. D., \& Lynskey, M. (2016). The increasing global health priority of substance use in young people. The Lancet Psychiatry, 3(3), 251-264.

10.- Chadi, N., Bagley, S. M., \& Hadland, S. E. (2018). Addressing Adolescents' and Young Adults' Substance Use Disorders. Medical Clinics of North America, 102(4), 603-620.

11.- Sigfúsdóttir, I. D., Thorlindsson, T., Kristjánsson, Á. L., Roe, K. M., \& Allegrante, J. P. (2009). Substance use prevention for adolescents: The Icelandic Model. Health Promotion International, 24(1), 16-25.

12.- Kristjansson, Alfgeir L., Sigfusdottir, I. D., \& Allegrante, J. P. (2013). Adolescent substance use and peer use: A multilevel analysis of cross-sectional population data. Substance Abuse: Treatment, Prevention, and Policy, 8(1), 1.

13.- Kristjansson, Alfgeir Logi, Sigfusson, J., Sigfusdottir, I. D., \& Allegrante, J. P. (2013). Data collection procedures for school-based surveys among adolescents: The youth in europe study. Journal of School Health, 83(9), 662667.

14.- Kristjansson, Alfgeir L., Sigfusdottir, I. D., Thorlindsson, T., Mann, M. J., Sigfusson, J., \& Allegrante, J. P. (2016). Population trends in smoking, alcohol use and primary prevention variables among adolescents in Iceland, 19972014. Addiction, 111(4), 645-652.

15.- Kristjansson, Alfgeir L., Mann, M. J., Sigfusson, J., Thorisdottir, I. E., Allegrante, J. P., \& Sigfusdottir, I. D. (2020a). Development and Guiding Principles of the Icelandic Model for Preventing Adolescent Substance Use. Health Promotion Practice, 21(1), 6269.

16.- Kristjansson, Alfgeir L., Mann, M. J., Sigfusson, J., Thorisdottir, I. E., Allegrante, J. P., \& Sigfusdottir, I. D. (2020b). Implementing the Icelandic Model for Preventing Adolescent Substance Use. Health Promotion Practice, 21(1), 70-79.

17.- Ortegón, E., Pacheco, J., \& Prieto, A. (2015). Adm Proy U2-2- Metodología del marco lógico para la planificación, el seguimiento y la evaluación de proyectos y programas. Retrieved from www.cepal.org/es/suscripciones

18.- Nilsson, T., Leifman, H., \& Andréasson, S. (2015). Monitoring local alcohol prevention in Sweden: Application of Alcohol Prevention Magnitude Measure (APMM). NAD Nordic Studies on Alcohol and Drugs, 32(5), 479-494.

19.- Bickel, W. K., Johnson, M. W., Koffar- 
nus, M. N., MacKillop, J., \& Murphy, J. G. (2014). The Behavioral Economics of Substance Use Disorders: Reinforcement Pathologies and Their Repair. Annual Review of Clinical Psychology, 10(1), 641-677.

20.- Acuff, S. F., Dennhardt, A. A., Correia, C. J., \& Murphy, J. G. (2019). Measurement of substance-free reinforcement in addiction: A systematic review. Clinical Psychology Review, 70(5), 79-90.

21.- Calafat, A., García, F., Juan, M., Becoña, E., \& Fernández-Hermida, J. R. (2014). Which parenting style is more protective against adolescent substance use? Evidence within the European context. Drug and Alcohol Dependence, 138(1), 185-192.

22.- Hemovich, V., Lac, A., \& Crano, W. D. (2011). Understanding early-onset drug and alcohol outcomes among youth: The role of family structure, social factors, and interpersonal perceptions of use. Psychology, Health \& Medicine, 16(3), 249-267.

23.- Hsiao, R. C. J., \& Walker, L. R. (2016). Understanding Adolescent Substance Use Disorders in the Era of Marijuana Legalization, Opioid Epidemic, and Social Media. Child and Adolescent Psychiatric Clinics of North America, 25(3), xiii-xiv.

24.- Olhaberry H., M., Crempien R., C., Biedermann C., K., Cruzat M., C., Martínez N., V., Martínez T., F., \& Krause J., M. (2011). Batería multidimensional de cuestionarios culturales para la investigación en salud mental: aplicación en una muestra pobalcional chilena. Revista Psiquiatría Clínica, 49(2), 9-21. Retrieved from http:// pesquisa.bvsalud.org/portal/resource/ pt/lil-702145

25.- Moreno, O., Janssen, T., Cox, M. J., Colby, S., \& Jackson, K. M. (2017).
Parent-adolescent relationships in Hispanic versus Caucasian families: Associations with alcohol and marijuana use onset. Addictive Behaviors, 74(January), 74-81.

26.- Mutumba, M., \& Schulenberg, J. E. (2019). Tobacco and Alcohol Use Among Youth in Low and Middle Income Countries: A Multi-Country Analysis on the Influence of Structural and Micro-Level Factors. Substance Use \& Misuse, 54(3), 396-411

27.- Marsiglia, F. F., Ayers, S. L., Han, S. Y., \& Weide, A. (2019). The Role of Culture of Origin on the Effectiveness of a Parents-Involved Intervention to Prevent Substance Use Among Latino Middle School Youth: Results of a Cluster Randomized Controlled Trial. Prevention Science, 20(5), 643-654.

28.- Marsiglia, F. F., Medina-Mora, M. E., Gonzalvez, A., Alderson, G., Harthun, M., Ayers, S., ... Kulis, S. (2019). Binational Cultural Adaptation of the keepin' it REAL Substance Use Prevention Program for Adolescents in Mexico. Prevention Science, 20(7), 1125-1135.

29.- Botvin, G. J., \& Griffin, K. W. (2004). Life skills training: Empirical findings and future directions. Journal of Primary Prevention, 25(2), 211-232.

30.- Velasco, V., Griffin, K. W., Botvin, G. J., Celata, C., Velasco, V., Antichi, M., ... Casalini, L. (2017). Preventing Adolescent Substance Use Through an Evidence-Based Program: Effects of the Italian Adaptation of Life Skills Training. Prevention Science, 18(4), 394-405.

31.- Jacobs, P., Estrada, Y. A., Tapia, M. I., Quevedo Terán, A. M., Condo Tamayo, C., Albán García, M., ... Prado, G. (2016). Familias Unidas for high risk adolescents: Study design of a cultural adaptation and randomized con- 
trolled trial of a U.S. drug and sexual risk behavior intervention in Ecuador. Contemporary Clinical Trials, 47, 244-253.

32.- Pérez-Gómez, A., Mejía-Trujillo, J., Brown, E. C., \& Eisenberg, N. (2016). ADAPTATION AND IMPLEMENTATION OF A SCIENCE-BASED PREVENTION SYSTEM IN COLOMBIA: CHALLENGES AND ACHIEVEMENTS. Journal of Community Psychology, 44(4), 538-545.

33.- Zamora, P. M., Fernanda, M., Rodríguez, R., Macías, F. C., Brown, E. C., Gómez, A. P., ... Toro, J. (2020). original adicciones vol. 32 , no $2 \cdot 2020$, 32(131), 105-115.
34.- Saxena, S., Paraje, G., Sharan, P., Karam, G., \& Sadana, R. (2006). The 10/90 divide in mental health research: Trends over a 10-year period. British Journal of Psychiatry, 188(1), 81-82.

35.- UNODC, \& WHO. (2018). International Standards on Drug Use Prevention - Second updated edition. United Nations Office on Drugs and Crime and World Health Organization, 58. Retrieved from http://www.unodc.org/documents/prevention/standards_180412. pdf
Correspondencia:

Carlos Ibáñez P

Santos Dumont, 999. Independencia, Región Metropolitana, Chile.

Código postal: 8380456

cibanez@hcuch.cl 